\title{
PAPER \\ Hand-Held System to Find Victims with Smartphones in Disaster Environment
}

\author{
Yasuyuki MARUYAMA ${ }^{\dagger}$, Nonmember and Toshiaki MIYAZAKI ${ }^{\dagger a}$, Senior Member
}

SUMMARY After a natural disaster it is critical to urgently find victims buried under collapsed buildings. Most people habitually carry smartphones with them. Smartphones have a feature that periodically transmits Wi-Fi signals called "Probe Requests" to connect with access points. Moreover, smartphones transmit "Clear to Send" when they receive a "Request to Send" alert. This motivated us to develop a hand-held smartphone finder system that integrates a novel method for accurately locating a smartphone using the Wi-Fi signals, to support rescue workers. The system has a unique graphical user interface that tracks target smartphones. Thus, rescue workers can easily reach victims who have their smartphones with them under collapsed buildings. In this paper, after introducing the localization method, the system architecture of the smartphone finder and its prototype system are described, along with some experimental results that demonstrate the effectiveness of the smartphone finder prototype.

key words: disaster, localization, received signal strength indicator, smartphone, Wi-Fi probe request

\section{Introduction}

Natural disasters, such as torrential rains and earthquakes, occur frequently worldwide [1]. When a building collapses owing to a disaster, rescue workers must urgently find the buried victims. Historically, smart aids such as fiberscopes [2] and rescue robots [3], [4] have been successfully used in rescue operations; however, these aids impose certain limitations.

It is now common for people to carry smartphones. The number of smartphone users is rising steadily, and is estimated to reach 3.8 billion in 2021, which is 1.52 times higher than in 2017 [5]. A smartphone sends out Wi-Fi signals called "probe requests" and "Clear to Send" (CTS), a motivating feature that could be effectively used to help the rescue workers estimate the locations of the victims in a disaster site such as a collapsed building. In this paper, we propose a smartphone finder (SF) that enables a rescue team to find buried victims quickly.

Figure 1 illustrates a scenario where victims with smartphones are buried under a collapsed building, and are found by rescue workers using the SF. The SF captures two types of Wi-Fi signals sent from the smartphone. When the received signals are adequate, the finder starts to estimate the location of the smartphone and course-corrects it by using the incoming signals. The estimated positions

\footnotetext{
Manuscript received March 18, 2020.

Manuscript revised June 15, 2020.

Manuscript publicized October 19, 2020.

The authors are with the University of Aizu, AizuWakamatsu-shi, 965-8580 Japan.

a)E-mail: miyazaki@u-aizu.ac.jp (Corresponding author) DOI: $10.1587 /$ transcom.2020EBP3040
}
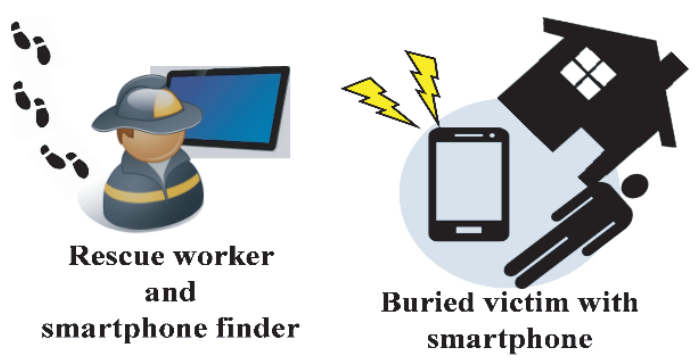

Fig. 1 Scenario of finding a buried victim with a smartphone using the smartphone finder.

are displayed on the screen of the finder, enabling the rescue workers to reach the smartphone and the victim. Note that we reported an initial version of this study [6]. However, a more detailed explanation of the proposed localization method and some limitations are added in this paper. Furthermore, we added a discussion on how to deal with "MAC address randomization," which is a privacy protection technique recently introduced in many smartphones.

The main contributions of this study are:

- A simplified packet sniffer is provided to collect and analyze the Wi-Fi signals from smartphones. It is functionally simple as it extracts only the information necessary for localization.

- MAC address randomization is studied to formulate measures against "MAC address randomization," which is an obstacle for finding appropriate smartphones.

- A novel localization method, which is a modified version of a conventional log-normal shadowing model, is developed to offer high accuracy using simple computations and suitable for use in disaster scenarios.

- A unique graphical user interface (GUI), which uses three types of GUI, is employed to easily capture the results.

\section{Related Works}

Localization methods using radio waves and their received signal strength indicator (RSSI) values have been proposed in various studies [7]-[11]. Zanella [7] introduced a method that considers the variability and accuracy of RSSI. Takashima et al. [8] proposed a method based on maximum likelihood (ML) that probabilistically estimates a position by using the distance relationship between RSSI and 
the target device; however, it requires pre-measurement of the environment and is, therefore, not applicable here. A similar method [9] that estimates positions using ML does not require pre-measurements but requires a sophisticated computer to perform complex computations. Thus, it is evident that the SF should be a hand-held PC or a tablet. In Refs. [10] and [11], the log-normal shadowing model is used for localization. It is widely used because it is simple and practical and estimates both the position of a target device and the propagation parameters by using simple computations. Therefore, we adopted the log-normal shadowing model here. However, the previous research projects usually did not consider real conditions, such as radio wave propagation and different obstacles in the disaster-hit area. In addition, they require the availability of unmanned aerial vehicles to be implemented. These are a few significant factors to be considered while adopting the localization methods as shown in previous studies.

\section{System Overview}

Figure 2 shows a system overview of the SF. It consists of a simplified packet sniffer, a localization function, and a unique set of GUIs that are realized as software components in a personal computer (PC). The GUI is connected to an offline map server, which does not require a network connection. A network interface card (NIC) is attached to the PC to capture Wi-Fi signals (Probe Request and CTS). Additionally, it is used to emit "Request to Send" (RTS) packets. Similarly, a GPS receiver attached to the PC helps to identify the location of the SF. The MAC address filtering function filters out unreliable data.

The following subsections detail the software components mentioned above.

\subsection{Simplified Packet Sniffer}

The sniffer captures the MAC address from the Wi-Fi proberequest and CTS signal, the complete frames of which are shown in Figs. 3 and 4, and logs it along with the RSSI value and acquisition time. In addition, the sniffer logs the location information of the SF, which is obtained by the GPS receiver. In comparison with the conventional packet sniffers such as Wireshark [12], our sniffer, which is simple and light extracts only the MAC address from the probe request frame and CTS frame. Figure 5 shows an example of the data captured by our packet sniffer.

Most smartphones can use both the 5-GHz and 2.4$\mathrm{GHz}$ band $\mathrm{Wi}-\mathrm{Fi}$; however, we used the 2.4-GHz band $\mathrm{Wi}-\mathrm{Fi}$ for localization, because the radio signal at lower frequencies is better for propagation. The path loss of radio wave signals in free space is often represented as follows [13]:

$$
\text { Pathloss }(d B)=32.5+20 \log F+\log d
$$

where $F$ is frequency [in $\mathrm{GHz}$ ] and $d$ is distance [in $\mathrm{m}$ ]. This formula indicates that the $2.4-\mathrm{GHz}$ band is better than the 5$\mathrm{GHz}$ band with regard to path loss.

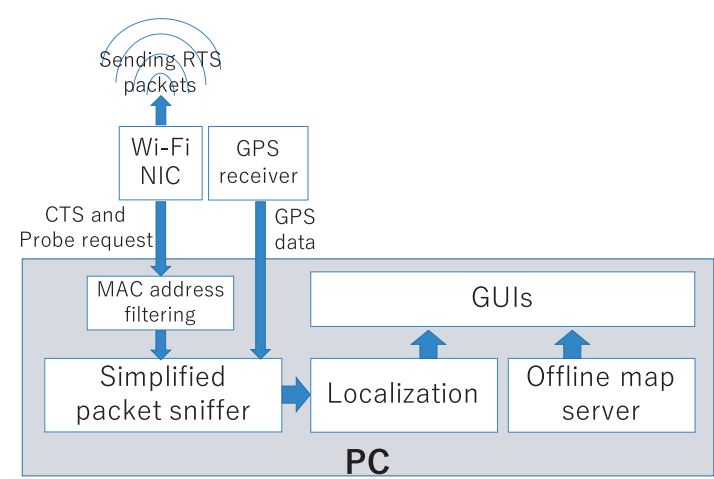

Fig. 2 System overview of the smartphone finder

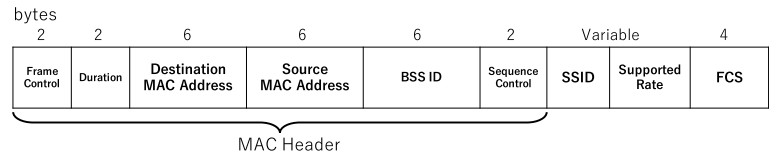

Fig. 3 Wi-Fi probe-request frame format.
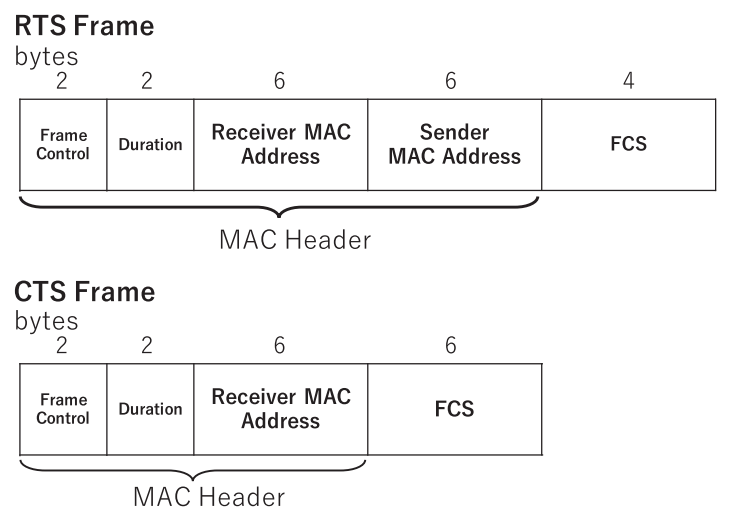

Fig. 4 RTS and CTS frame formats.

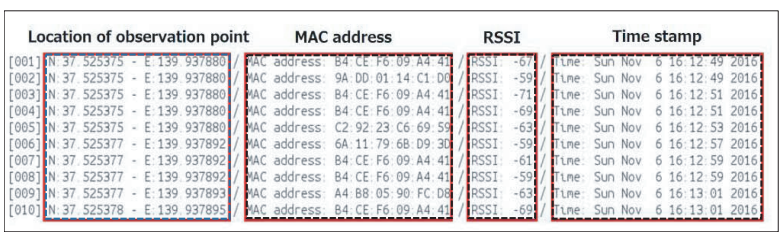

Fig.5 Example of captured packets.

To confirm the actual path loss, we conducted several preliminary experiments. Figure 6 shows the RSSI experimental results plotted against the distance between a sniffer and a smartphone in an open space environment. In this experiment, the sniffer was carried by a person, while the target smartphone was placed $1 \mathrm{~m}$ above the ground on a table. In the experiment, data having the same RSSI values were observed at a distance. To represent the quantity of data obtained at the same point, different colors are used in the figure. Large dots represent averaged values at the corresponding distance. The standard deviation is also drawn using a line at each averaged value. 


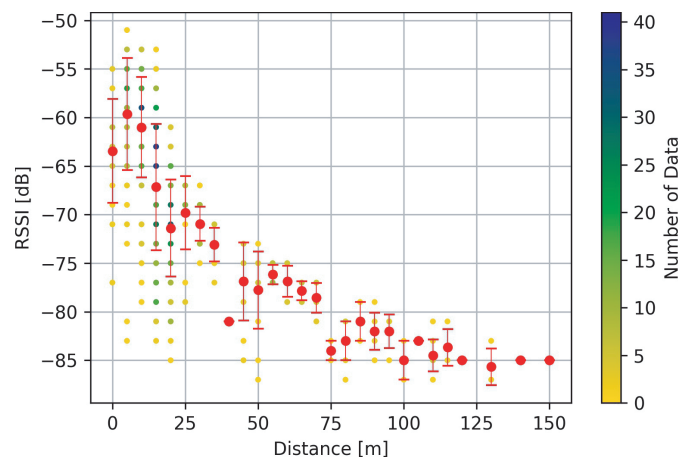

Fig. 6 Relationship between RSSI and distance in an open space environment.

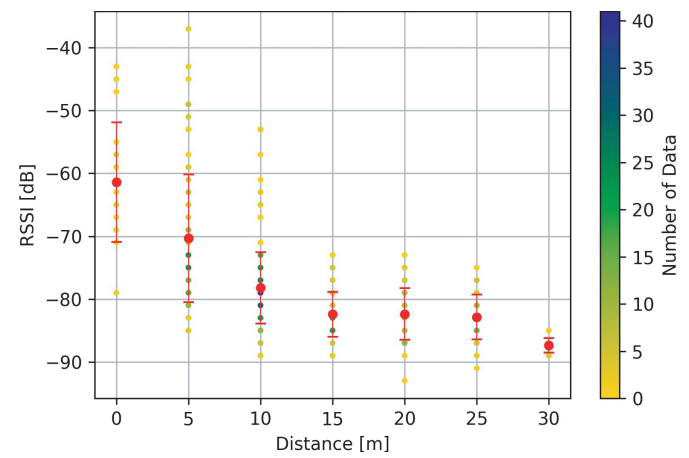

Fig. 7 Relationship between RSSI and distance in an adverse environment.

Smartphones are generally in the pockets or bags of their owners, approximately $1 \mathrm{~m}$ above the ground. Therefore, in this experiment, we placed the smartphone at a height of $1 \mathrm{~m}$. For each distance, we measured the RSSI values for $1 \mathrm{~min}$. In the figure, the small dots indicate the observed RSSI values, whereas the large dots indicate the average of the observed values at each distance. Although, we failed to obtain the RSSI values in some cases owing to situational factors, and the number of values was found to vary with distance; the experimental results confirmed the satisfactory operation of our sniffer.

Figure 7 shows the experimental results obtained in an adverse environment analogous to a disaster. The target smartphone was placed on the ground and covered with obstacles. This is assumed to be a scenario in which the smartphone is under a collapsed building with its owner. Compared to Fig. 6 (open space environment), both the number of probe requests acquired and the maximum reachable distance decreased and the variation in RSSI increased. This indicates that we cannot use the obtained RSSI values to estimate the distance between the SF and the target smartphone, which is elaborated in the proposed localization method section.

There are two types of Wi-Fi antennas: directional and non-directional. Although the directional antenna is powerful in specific directions, it has a high implementation cost as the location estimation needs a geomagnetic sensor and extra calculations. Moreover, as we were focused on devising a small and portable system, we preferred to use a non-directional antenna for our sniffer.

\subsection{MAC Address Randomization and Its Partial Solution}

As previously stated, the MAC address is used to identify the smartphone/tablet in our system. The MAC address is a unique identifier that all 802.11 devices have. Unfortunately, this can create privacy issues because third parties can identify individual traffic and devices. In particular, the probe request frames are continuously broadcasting, making them vulnerable to monitoring and tracking. To solve this problem, some devices may use a temporary, randomized MAC address that changes periodically and is different from the true MAC address. Consequently, a third party cannot track the device using the MAC address. This effectively solves privacy issues, however, if this mechanism always works, we cannot identify the device even in a disaster scene.

Today, most mobile devices including Android and iOS smartphones have the MAC address randomization mechanism [14]. In addition, whether the randomization is enabled or not depends on the operating systems and versions. In [15], it was reported that MAC address randomization is not complete from the privacy protection perspective and allows for a device identification method. However, additional computation power and storage devices are required to implement the proposed device identification method. Thus, it is difficult to introduce this method in our SF.

However, we found a solution to the MAC address randomization problem in the RTS/CTS transmissions. RTS/CTS transmissions are a mechanism used in the 802.11 wireless networking protocol to reduce frame collisions caused by hidden node problems. When a terminal requests data from other terminals, it sends an RTS packet. Next, when the other terminal receives the RTS packet, it sends back a CTS as an acknowledgment of the request. Then, a connection between the terminals is established to exchange data. The flaw in RTS/CTS is when RTS is sent to a correct target device's MAC even if it uses a randomized MAC address a CTS with the true MAC address is returned. The cause of this behavior is that the RTS/CTS response is not a function of the operating system (OS), but a function of the IEEE 802.11 chipset. Thus, this problem cannot be fixed with a simple patch or OS update. By using this flaw, we implemented the following function in the SF.

1. The CTS response has the property of emitting only when RTS is transmitted to the true MAC address. Therefore, sending an RTS packet to the MAC address of the packet observed by the sniffer can check whether the MAC address is randomized or correct. Then, the SF filters randomize the MAC addresses based on the result and only the true MAC addresses are saved.

2. After obtaining a valid MAC address, the SF transmits 
Table 1 Tablet devices for verification and experiment.

\begin{tabular}{cc}
\hline \hline Manufacturer & Operating System \\
\hline Huawei & Android 6.0.1 \\
ASUS & Android 7.0 \\
Google & Android 6.0.1 \\
Apple & iOS 10.3.4 \\
\hline \hline
\end{tabular}

RTS to the true MAC address periodically, and it obtains CTS packets from the device that has the MAC address. The collected CTS packets are used to estimate the location of the device by applying the method described in 3.3.

This function is implemented in our SF, and is verified using tablet devices shown in Table 1. Although there is a difference in the number of packets observed depending on the device types and settings, we confirmed that the CTS packets sent from the devices carry valid MAC addresses.

\subsection{Proposed Localization Method}

We considered a target smartphone located at $(\hat{x}, \hat{y})$ and the RSSI value $P_{i}$ of radio waves from the SF are observed at $\left(x_{i}, y_{i}\right)$. Let $d_{i}$ be the distance between $(\hat{x}, \hat{y})$ and $\left(x_{i}, y_{i}\right)$. In the log-normal shadowing model [9], [10], $P_{i}$ is theoretically calculated as follows:

$$
\begin{aligned}
\widehat{P}_{i} & =\widehat{P_{d_{0}}}-10 \hat{n} \log _{10}\left(\frac{d_{i}}{d_{0}}\right) \\
\mathrm{d}_{\mathrm{i}} & =\sqrt{\left(x_{i}-\hat{x}\right)^{2}+\left(y_{i}-\hat{y}\right)^{2}}
\end{aligned}
$$

where $d_{0}$ is a reference distance that equals $1[\mathrm{~m}], \hat{P}_{d_{0}}$ is an RSSI value observed at $d_{0}$, and $\hat{n}$ is a path loss exponent. For the conventional localization method, we calculate the leastsquares as below using the experimental $P_{i}$ and the theoretical $\hat{P}_{i}$ values obtained from Eq. (1) for $\theta$, which includes the location of the target smartphone and propagation parameters:

$$
\begin{aligned}
& \min _{\theta} \sum_{i}\left(P_{i}-\hat{P}_{i}\right)^{2} \\
& \text { s.t.: } \quad \theta=\left\{(\hat{x}, \hat{y}), \hat{P}_{d_{0}}, \hat{n}\right\}
\end{aligned}
$$

Here, we consider the "reliability" of the measured radio waves (RSSI values). They show greater reliability as their RSSI values are high and increase with distance. Considering this, an appropriate weight is introduced to each term in the conventional least-squares method, and the resultant weighted least-squares are obtained as follows.

$$
\begin{aligned}
& \min _{\theta} \sum_{i} \frac{P_{i}}{P_{\max }}\left(P_{i}-\hat{P}_{i}\right)^{2} \\
& \text { s.t.: } \\
& \theta=\left\{(\hat{x}, \hat{y}), \hat{P}_{d_{0}}, \hat{n}\right\}, \\
& 2 \leq \hat{n} \leq 4, \text { and }
\end{aligned}
$$

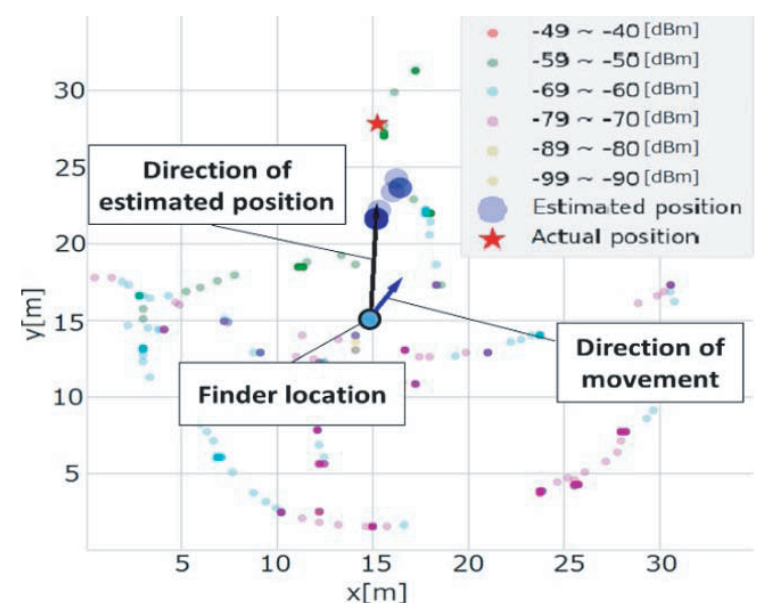

Fig. 8 A screenshot of the first GUI for navigating the user of the target smartphone.

$$
-50<\hat{P}_{d_{0}}<-48
$$

where $P_{\max }$ is the largest value among the observed RSSI values. The estimation error is minimized by providing additional constraints (7) and (8) where the numbers are based on the data obtained in preliminary experiments. Equation (7) represents the pass-loss level. If $\hat{n}=2$, the radio propagation environment is adjacent to open space, but in the case of $\hat{n}=4$ or more, radio reception cannot be expected. $\hat{P}_{d_{0}}$ is the RSSI value at $1 \mathrm{~m}$ from the target smartphone. The value range represented by Eq. (8) is determined by the preliminary experiments. By adding the constraints (7) and (8), we can eliminate unrealistic estimation results. In addition, the introduction of the constraints contributes to reducing the estimation time. This is because $\theta$ (i.e., four parameters) must be calculated simultaneously, and the constraints narrow the solution space that has to be searched. This is our proposed localization method. By replacing Eqs. (3)-(4) with Eqs. (5)-(8), we can estimate the location of the target smartphone with high accuracy.

\subsection{Proposed Graphical User Interface}

A screenshot of the GUI of the SF that is critical to achieving the rapid rescue of victims is shown in Fig. 8. In the figure, the blue directional arrow indicates the movement of the SF, whereas the black directional arrow indicates the estimated position of a target smartphone. If the rescue worker selects the estimated position of one smartphone, the smartphone is locked on, and the black navigation line is displayed between the target smartphone and the finder. Thus, by following this navigation line, the worker can find the smartphone together with the victim easily. If the target smartphone is lost because of missing packets, the blue navigation line will disappear or freeze on the screen. In this case, the rescue worker must select another smartphone as the new target. Here, we cannot select two or more target smartphones simultaneously. Moreover, each small dot shows the past trajectory of the SF, and its color represents the observed RSSI 


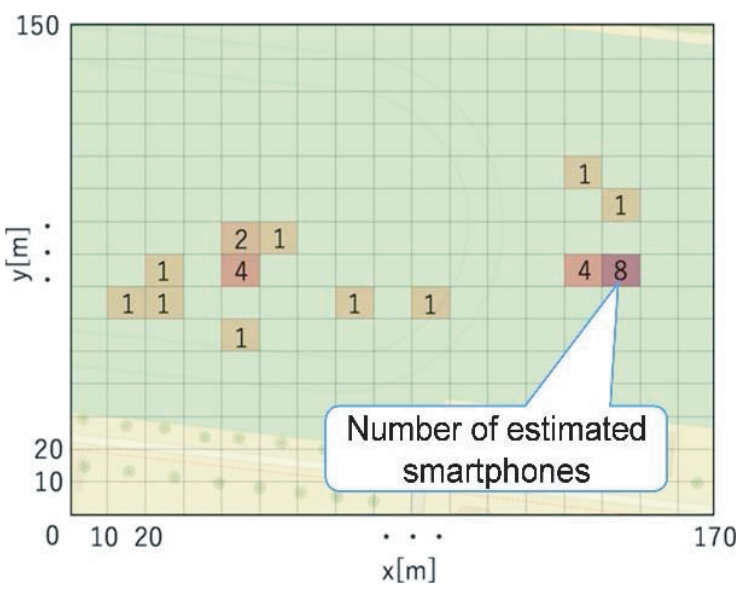

Fig. 9 A screenshot of the second GUI for displaying multiple smartphones.

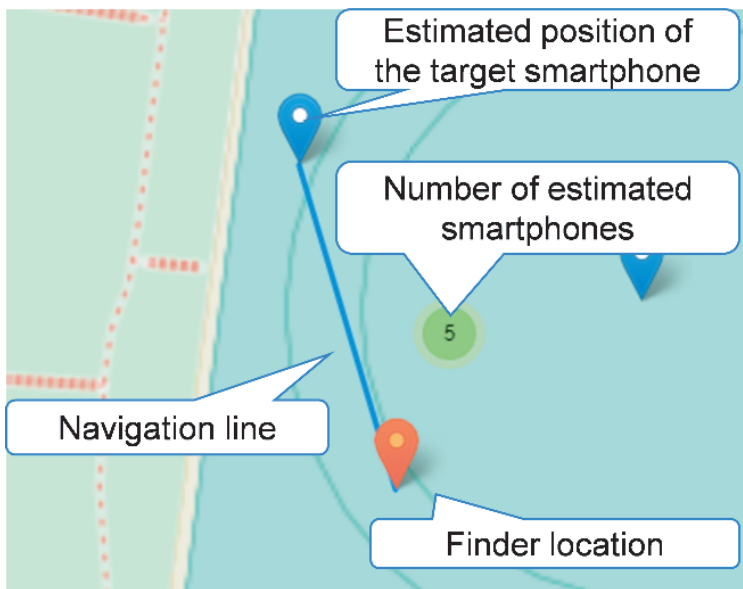

Fig. 10 A screenshot of the third GUI for displaying the target and multiple smartphones.

value at that point. The color changes from a light color to red depending on the RSSI value. By checking this information on the SF, the rescue worker can navigate to the victim who is expected to be near the target smartphone.

In a real-time disaster site, there could be many people with smartphones making it critical for multiple smartphone locations to be displayed to facilitate an appropriate assessment of the situation. Therefore, we developed two types of GUI, the schematics of which are shown in Figs. 9 and 10. In Fig. 9, the site area is divided into 10-m meshes, and the number of estimated smartphones is displayed in the corresponding mesh. Depending on the number of smartphones in it the color of the mesh changes. The dark red color indicates the presence of many smartphones in the area. By using this GUI, the rescue worker can recognize priority areas.

Another GUI that reflects multiple smartphones is shown in Fig. 10. In this figure, the red marker indicates the current location of an SF, whereas the blue marker indicates the estimated position of the smartphone. The color of the navigation line displayed between the target smartphone

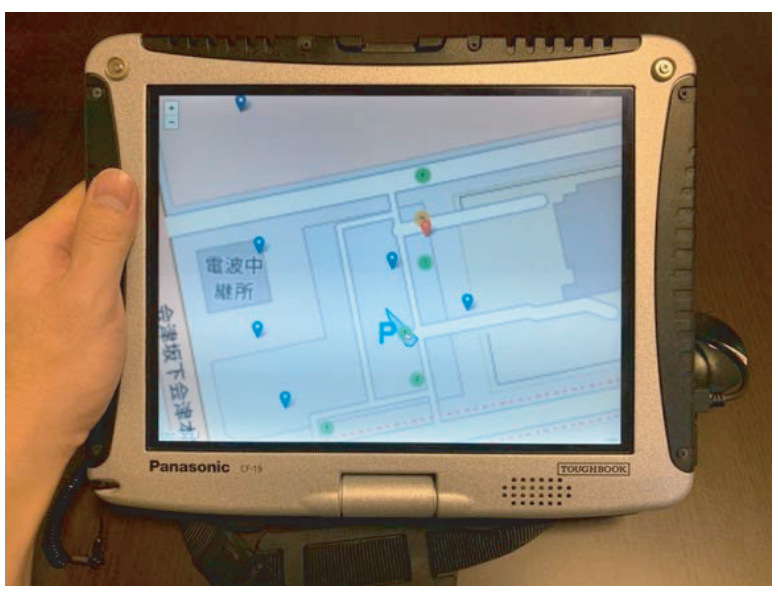

Fig. 11 Prototype system of the smartphone finder.

and the finder is blue in this GUI. Moreover, the number " 5 " on the green circle in this schematic indicates the number of smartphones identified in that area. If the circle is clicked, detailed information would be displayed on the screen. This GUI is simple as it helps identify the estimated position of the smartphone by a simple online display of appropriate information. This is an upgraded version of the GUI shown in Fig. 8, as it is found to be relatively more effective particularly in the event of many smartphones being in the search area.

Thus, we proposed three different GUIs thereby enabling the user to switch from one GUI to another easily depending on the situation. To produce the GUIs, we used "OpenStreetMap" [16] with an open-source library using JavaScript (namely "Leaflet") [17] for interactive maps, and several other resources. A large-scale disaster often damages the infrastructure such as internet and telecommunication networks. In such cases, internet-based map services like Google Maps cannot be used [18]. Thus, we introduced OpenStreetMap that supports offline map services when installed in a local map server, and the SF can display a map at any time on each of the GUIs without an internet connection.

\subsection{Prototype System}

We developed a prototype system of the SF, a snapshot of which is shown in Fig. 11. We used a small, lightweight Linux PC with a touch panel (Panasonic TOUGH BOOK CF-19, OS: Ubuntu 16.04) to realize the functions of the simplified packet sniffer, localization, and unique GUIs. In addition, it was equipped with an external network interface card (NIC) (WLI-UC-GNME) for packet capture and RTS transmission. The NIC had a non-directional antenna and supported a monitor mode, which was required to handle the raw packets received from the application layer to realize the sniffer function. Moreover, a GPS receiver (Globalsat BU 353 S4) [19] was connected to the PC using a USB interface to capture the location of the SF. The data sampling rate of the GPS was $2.4 \mathrm{KHz}$. The GPS reacquisition 
time was $0.1 \mathrm{~s}$ on average and the localization accuracy was $2.5 \mathrm{~m}$ 2DRMS (twice the distance root mean square), that is, there was a 95-98\% probability that the location was within a $2.5-\mathrm{m}$ radius. The simplified packet sniffer was developed using C and the "libpcap" library [20] to capture and analyze the Wi-Fi probe requests and CTS. The RTS transmission and MAC address filtering were developed using Python and the SCAPY packet crafting tool [21]. The sniffer extracts only the information necessary for the localization function that was developed using Python and a mathematics package, namely, "SciPy" [22] for estimating the locations of the target smartphones. Location estimation was performed for each smartphone individually by using the captured data and a smartphone was identified using the captured MAC address. In this way, location estimation was achieved for multiple smartphones by applying the proposed localization method to each device. The unique GUIs were developed using Python and JavaScript.

Here, the acquisition times for locating the SF by GPS, and obtaining the target smartphone's location were different, and to synchronize these timings is difficult because the time required for packet sniffing is not controllable. This could cause errors in determining locations. However, we assume that the SF is carried by a rescue worker and that he/she moves at a walking speed, i.e., approximately $1.5 \mathrm{~m} / \mathrm{s}$. In addition, assuming that the target smartphone is with a victim under collapsed buildings, it will probably be static. Under these assumptions, we believe that it is not a serious problem, because the expected location error is not changed even if the time synchronization is not realized.

Rescue workers could accept the SF as a victim-finding aid, even if its location estimation error is $10 \mathrm{~m}$ or more from a victim. This is because they could identify a search area in a disaster scene from prior information and by applying their judgment.

\section{Performance Evaluation}

The localization performance was evaluated using the prototype system. The experimental conditions were as follows:

- Experiment location: an open space on our university campus (outdoor).

- Trials: seven were conducted (272 to 559 packets were acquired per trial.)

- Experimental procedure: A smartphone was placed on the ground and covered with obstacles (Clothes, Cardboard, or Iron).

- Target devices: Smartphones/Tablets shown in Table 1 were used for the evaluations.

For the experiment, an assistant randomly walked around the smartphone for $10 \mathrm{~min}$ with the SF and captured Wi-Fi packets. For comparison purposes, we also evaluated a conventional localization method based on the log-normal shadowing model using the same acquired data.

Table 2 shows the comparative results of our proposed method with a conventional localization method based on
Table 2 Comparison of conventional and proposed method.

\begin{tabular}{ccc}
\hline \hline Method & Averaged error (m) & Standard deviation (m) \\
\hline Conventional & 36.1 & 41.6 \\
Proposed & 7.6 & 5.0 \\
\hline \hline
\end{tabular}

Table 3 Comparison of the estimation error in the conventional and proposed methods for each obstacle.

\begin{tabular}{lcc}
\hline \hline Obstacle & $\begin{array}{c}\text { Conventional method } \\
(\mathrm{m})\end{array}$ & $\begin{array}{c}\text { Proposed method } \\
(\mathrm{m})\end{array}$ \\
\hline Clothes & 6.5 & 5.7 \\
Cardboard & 47.5 & 9.9 \\
Two cardboards & 34.4 & 12.5 \\
Iron & 114.6 & 12.8 \\
None & 1.2 & 1.1 \\
\hline \hline
\end{tabular}

the log-normal shadowing model with regard to the average and standard deviation of the estimation error. It is evident that the proposed method performs the localization with significantly higher accuracy compared to the conventional method. The result also implies that a smartphone can be discovered by searching in an area with a radius of approximately $13 \mathrm{~m}(7.6 \mathrm{~m}+5.0 \mathrm{~m})$ around the estimated position.

Table 3 compares the estimation error in the proposed and conventional methods for each type of debris that was used to cover the target smartphone. Here, we introduced three different kinds of obstacle (clothes, cardboard, and iron), to replicate the scenario of victims being under a collapsed building near their smartphones. In all cases, our proposed method's estimation error was low, and our method over-performed in the case of iron. This clearly establishes that our localization method is suitable for tracing smartphones under collapsed buildings.

Note that the significantly different number of packets acquired arose because the radio signal from the smartphones/tablets was weakened. This was caused by the debris and differences in the probe packets transmission timings of the smartphones/tablets shown in Table 1. This happened despite our location estimation model having introduced some real constraints by using Eqs. (7) and (8), but showing much better performance than a conventional one as shown in Tables 2 and 3.

Figure 12 shows plots of an estimation result where the star ( $\star$ ), disc (○), and small dots indicate the actual position of the smartphone, the estimated position, and the past trajectory of the SF, respectively. This result too proves the ability of our SF efficiently to locate the target smartphones.

\section{Limitations and Expected Usage of Smartphone Finder}

If we consider a real disaster situation, some limitations of 


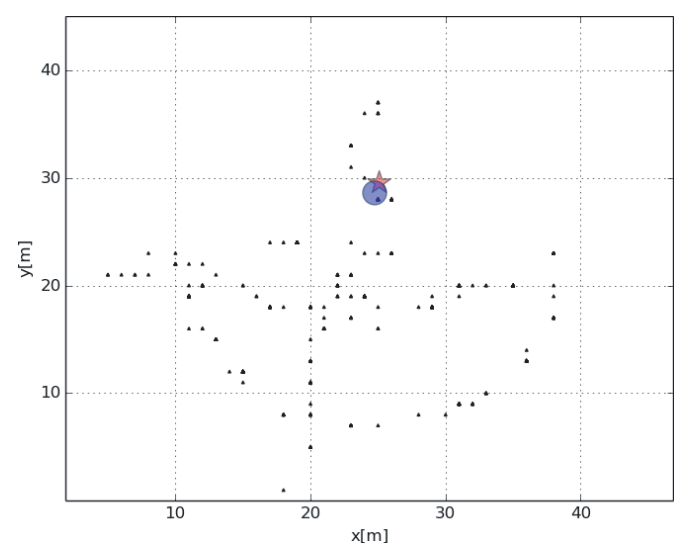

Fig. 12 Plots of the experimental results.

our SF are listed below:

- How quickly it finds a smartphone depends on how often the smartphone sends the probe request frames. Almost all recent smartphones have a power-saving mode, and this may extend the smartphone's probe request packets' time interval.

- Although a packet filtering function to eliminate captured packets using randomized MAC addresses was implemented, the MAC address randomization problem discussed in Sect. 3.4 was not solved completely. Thus, our SF may estimate the number of smartphones in the target area to be more than the actual number.

Despite these observations we believe that our SF is useful as an aid to finding victims in a disaster area. This is because rescue workers often identify which debris fields and landslide areas to search by using their experience, judgment, and other information. However, the presence of smartphones and their locations obtained by our SF could help them to narrow the search zones, and prioritize searches, even if the smartphone information is uncertain and/or overestimated.

\section{Conclusions}

In this paper, we proposed a smartphone finder (SF) to rescue victims buried with their smartphones under collapsed buildings or similar obstacles in a disaster site. A simplified packet sniffer was developed to capture and analyze the Wi-Fi signals transmitted from their smartphones. It only extracts the information necessary for location estimation and is, therefore, simpler than general-purpose sniffers. We also investigated a flaw in the MAC address randomization mechanism used in many mobile devices and implemented a function to filter the packets containing randomized MAC addresses. To determine the location of a target smartphone with high accuracy, a new method was proposed that considers the reliability of the obtained RSSI values. The experimental results demonstrate that the accuracy of our proposed method is $7.6 \mathrm{~m}$, which is better than the conventional method to which it was compared. Further- more, three unique GUIs were developed to help the rescue workers find the smartphones easily. By moving around a disaster-hit area with the proposed SF, the workers can find the victims with smartphones easily even if they are buried under collapsed buildings. We firmly believe that our SF can contribute to rescue operations in natural disasters because it has become a common practice for people always to carry smartphones.

\section{Acknowledgments}

This work was partly supported by the Council for Science, Technology and Innovation (CSTI), the Cross-ministerial Strategic Innovation Promotion Program (SIP), and "Enhancement of societal resiliency against natural disasters" (Funding agency: JST).

\section{References}

[1] Statista Research Department, "Natural disasters - Statistics \& facts," Jan. 28, 2020. [Online]. Available: https://www.statista.com/ topics/2155/natural-disasters/

[2] K. Hatazaki, M. Konyo, K. Isaki, S. Tadokoro, and F. Takemura, "Active scope camera for urban search and rescue," IEEE/RSJ International Conference on Intelligent Robots and Systems, San Diego, CA, USA, pp.2596-2602, Dec. 2007.

[3] Y. Liu and G. Nejat, "Robotic urban search and rescue: A survey from the control perspective," J. Intell. Robot. Syst., vol.72, no.2, pp.147-165, March 2013.

[4] B. Shah, and Howie Choset, "Survey on urban search and rescue robots,” J. Robot. Soc. Jpn., vol.22, no.5, pp.582-586, July 2004.

[5] Statista, S. O'Dea, "Number of smartphone users worldwide from 2016 to 2021 (in billions)," Feb. 28, 2020. [Online]. Available: https://www.statista.com/statistics/330695/number-ofsmartphone-users-worldwide/

[6] Y. Maruyama and T. Miyazaki, "Smartphone finder: Dedicated to seeking victims under collapsed buildings," 13th International Conference on Ubiquitous Information Management and Communication (IMCOM2019), Phuket, Thailand, pp.517-528, Jan. 2019. DOI: 10.1007/978-3-030-19063-7_40

[7] A. Zanella, "Best practice in RSS measurements and ranging," IEEE Commun. Surveys Tuts., vol.18, no.4, pp.2662-2686, Oct. 2016. DOI: $10.1109 /$ COMST.2016.2553452

[8] M. Takashima, D. Zhao, K. Yanagihara, K. Fukui, S. Fukunaga, S. Hara, and K. Kitayama, "Location estimation using received signal power and maximum likelihood estimation method in wireless sensor networks," Electron. Commun. Jpn., vol.90, no.12, pp.62-72, June 2007. DOI: 10.1002/ecja.20359

[9] M. Zemek and S. Hara, "A novel target localization without a prior knowledge on channel model parameters," IEICE Technical Report, vol.107, no.81, pp.13-18, May 2007.

[10] J. Sundqvist, J. Ekskog, B.J. Dil, F. Gustafsson, J. Tordenlid, and M. Petterstedt, "Feasibility study on smartphone localization using mobile anchors in search and rescue operations," 19th International Conference on Information Fusion (FUSION), Heidelberg, pp.1448-1453, 2016.

[11] B.J. Dil and P.J.M. Havinga, "RSS-based self-adaptive localization in dynamic environments," 3rd IEEE International Conference on the Internet of Things, Wuki, pp.55-62, 2012.

[12] Wireshark, 2018. Available: https://www.wireshark.org/

[13] M. Gast, 802.11 Wireless Networks: The Definitive Guide, 2nd ed., O’Reilly Media, 2005.

[14] J. Martin, T. Mayberry, C. Donahue, L. Foppe, L. Brown, C. Riggins, E.C. Rye, and D. Brown, "A study of MAC address random- 
ization in mobile devices and when it fails," Proc. Privacy Enhancing Technologies, no.4, pp.268-286, March 2017. DOI: 10.1515/ popets-2017-0054

[15] M. Vanhoef, C. Matte, M. Cunche, L.S. Cardoso, and F. Piessens, "Why MAC address randomization is not enough: An analysis of Wi-Fi network discovery mechanisms," 11th ACM on Asia Conference on Computer and Communications Security, May 2016, pp.413-424. DOI: 10.1145/2897845.2897883

[16] "OpenStreetMap: A free wiki world map," Available: https://www. openstreetmap.org/

[17] "Leaflet: an open-source JavaScript library for interactive maps," Available: https://leafletjs.com/

[18] "Google map: A web mapping service developed by Google," Available: https://www.google.com/maps

[19] "GPS Mouse User's Guide V1.0 (WIN)," Available: https://www. globalsat.com.tw/ftp/download/GMouse_Win_UsersGuide-V1.0.pdf

[20] "Libpcap: A portable $\mathrm{C} / \mathrm{C}++$ library for network traffic capture," Available: https://www.tcpdump.org/

[21] "SCAPY: Packet crafting for Python2 and Python3," Available: https://scapy.net

[22] "SciPy: Open source scientific tools for Python," Available: http:// www.scipy.org/

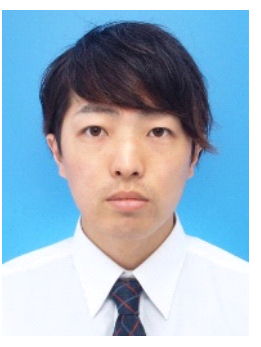

Yasuyuki Maruyama was born in Japan in 1995. He received the B.S. and M.S. degrees in computer science and engineering from The University of Aizu, Fukushima, Japan, in 2018, and 2020. His research interests include the localization of smartphones using Wi-Fi signals for a disaster scene and its system development.

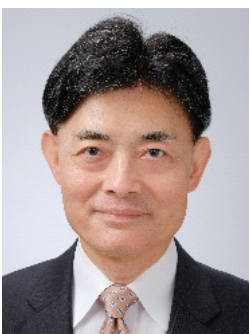

Toshiaki Miyazaki is the Chairperson and President of the University of Aizu, Fukushima, Japan. His research interests are in adaptive networking technologies, reconfigurable hardware systems, and autonomous systems. $\mathrm{He}$ received the B.E. and M.E. degrees in applied electronic engineering from the University of Electro-Communications, Tokyo, Japan in 1981 and 1983, and the Ph.D. degree in electronic engineering from Tokyo Institute of Technology in 1994, respectively. Before joining the University of Aizu, he worked for NTT for 22 years, and engaged in research on VLSI CAD systems, telecommunications-oriented FPGAs and their applications, active networks, peer-to-peer communications, and ubiquitous network environments. Dr. Miyazaki was a visiting professor at the graduate school, Niigata University in 2004, and a part-time lecturer at the Tokyo University of Agriculture and Technology from 2003-2007. He has published more than 350 technical papers including IEEE Transactions and conferences and has 50 patents. He has served as a general/program chair or program committee member at many international conferences. These include IEEE MCSoC and IEEE CIT. He is a senior member of IEEE, IEICE, and IPSJ. 